\title{
COMPLICACIONES DE LA VENTILACIÓN MECÁNICA EN EL POSOPERATORIO DE CIRUGÍA CARDIOVASCULAR
}

\section{COMPLICATIONS OF MECHANICAL VENTILATION IN POSTOPERATIVE CARE OF CARDIOVASCULAR SURGERY}

\author{
FERNANDO RÍOS', NICOLÁS DIEZ²
}

\section{RESUMEN}

La cirugía cardíaca conlleva una variedad de complicaciones pulmonares, algunas de las cuales contribuyen a la lesión pulmonar o síndrome de dificultad respiratoria aguda, y se asocia con aumento de la permeabilidad vascular pulmonar, el incremento del agua pulmonar extravascular y/o la generación de atelectasias. Los mecanismos implicados en el desarrollo de estas incluyen dos causas fundamentales: el trauma del procedimiento quirúrgico y el efecto de la ventilación mecánica sobre un entorno inflamatorio. Esta revisión se enfocará en abordar las diferentes efectos cardiovaculares y complicaciones de la ventilación mecánica en pacientes en el posoperatorio de cirugía cardíaca, y además se revisarán diferentes estrategias terapéuticas para minimizar la lesión pulmonar.

Palabras clave: respiración artificial; intubación; cirugía torácica.

\section{ABSTRACT}

Cardiac surgery involves a variety of pulmonary complications, some of which contribute to lung injury or acute respiratory distress syndrome, and is associated with increased pulmonary vascular permeability, increased extravascular pulmonary water, and / or the generation of atelectasis. The mechanisms involved in the development of these include two fundamental causes: the trauma of the surgical procedure and the effect of mechanical ventilation on an inflammatory environment. This review will focus on addressing the different cardiovascular effects and complications of mechanical ventilation in patients in the postoperative period of cardiac surgery, and will also review different therapeutic strategies to minimize lung injury.

Keywords: respiration; intubation; thoracic surgery.

REVISTA CONAREC 2018;33(146):221-225 | DOI:10.32407/RCON/2018146/0221-0225

\section{INTRODUCCIÓN}

La ventilación mecánica (VM) también se denomina ventilación con presión positiva. Consiste en la entrega de una mezcla predeterminada de gases (oxígeno y otros gases) de manera forzada en las vías respiratorias centrales que luego fluye hacia los alvéolos. A medida que estos gases ingresan a los pulmones durante la inspiración, la presión intraalveolar aumenta. La espiración se produce pasivamente liberando el gas entregado por diferencia de presión entre la vía aérea y la atmósfera. Los principales beneficios de la VM son la mejora del intercambio de gases y la disminución del trabajo respiratorio; mejora el intercambio de gases al mejorar la relación ventilación-perfusión (V/Q). El trabajo respiratorio puede aumentar debido a la alteración de la mecánica pulmonar (p. ej., aumento de la resistencia de las vías respiratorias o disminución de la compliance) o por aumento de la demanda respiratoria (p. ej., acidosis, dolor, etc.). El esfuerzo requerido para mantener este trabajo respiratorio elevado puede ocasionar fatiga de los músculos respiratorios e insuficiencia respiratoria. La VM

1. Jefe de Terapia Intensiva en Sanatorio Las Lomas. Staff de Terapia Intensiva de Adultos, Hospital Nacional Alejandro Posadas.

2. Residente de Terapia Intensiva de Adultos, Hospital Nacional Alejandro Posadas.

$\triangle$ Correspondencia:Dr. Fernando Ríos. conarecrevista@gmail.com

Los autores declaran no tener conflictos de intereses.

Recibido: 06/06/2018| Aceptado: 01/08/2018 puede asumir parte o todo el trabajo respiratorio, permitiendo que los músculos ventilatorios se recuperen.

La cirugía cardíaca conlleva una variedad de complicaciones pulmonares, algunas de las cuales contribuyen a la lesión pulmonar o síndrome de dificultad respiratoria aguda (SDRA). La cirugía cardíaca se asocia con aumento de la permeabilidad vascular pulmonar, el incremento del agua pulmonar extravascular (EVLW) y/o la generación de atelectasias. Estas alteraciones pueden actuar solas o en conjunto, y pueden contribuir a las anormalidades mecánicas o del intercambio gaseoso, como el desarrollo de hipoxemia y dependencia temporal del ventilador. También pueden estar relacionadas a procesos inflamatorios de origen múltiple, como la circulación extracorpórea, el daño por isquemia-reperfusión, la presencia de derrame pleural y la ventilación mecánica per se o daño inducido por la ventilación mecánica.

\section{VENTILACIÓN MECÁNICA Y CIRUGÍA CARDÍACA}

En un adulto sano normal en posición vertical, la capacidad residual funcional (CRF) es aproximadamente de 3 litros y disminuye en 0,70,8 litros en la posición supina cuando el contenido abdominal empuja el diafragma en dirección cefálica. Esto se acentúa aún más con la anestesia y la parálisis, ya que la presión abdominal se transmite fácilmente a la cavidad torácica. Luego de la cirugía cardiovascular, la CRF se reduce un 40\% en contraste al 20\% observado en el resto de los posoperatorios ${ }^{1,2}$. Si bien no está clara la razón para esta diferencia, varios factores parecen contribuir y confluir en un evento inflamatorio pulmonar. La circulación extracorpórea (CEC), la injuria induci- 
Tabla 1. Efecto cardiovascular de la ventilación mecánica. PVC: presión venosa central. VD: ventrículo derecho. VI: ventrículo izquierdo.

\section{Cambios generados por la ventilación mecánica}

Incremento en la presión intratorácica.

Disminución del volumen sanguíneo pulmonar y aumento del volumen sanguíneo sistémico.

Disminución del retorno venoso.

Disminución del volumen sistólico del VD.

Disminución en la presión arterial pulmonar.

Disminución en las presiones de llenado de los ventrículos.

Disminución del volumen sistólico del VI.

\section{Comentario}

La presión positiva aplicada a los pulmones provoca la compresión del parénquima pulmonar contra la pared del tórax

Durante la fase inspiratoria de la ventilación de presión positiva, una fracción del volumen sanquíneo pulmonar se desplaza a la circulación sistémica. Esto no aumenta la PVC, porque la circulación venosa sistémica puede absorber fácilmente este volumen extra.

La mayor presión intratorácica impide el retorno venoso sistémico de sangre al VD.

La disminución del retorno venoso al VD conduce a la disminución de su volumen sistólico.

Disminución del volumen sistólico del VD reduce el volumen sanguíneo (presión) en las arterias pulmonares.

La disminución del volumen sistólico del VD y la caída de la presión de la arteria pulmonar y provoca una reducción de la precarga y del volumen sistólico del VI. da por la isquemia-reperfusión y la VM juegan un rol importante en el proceso inflamatorio pulmonar posquirúrgico. Se han observado reducciones marcadas en el FEV 1 y la capacidad vital forzada (CVF) hasta 5 días después de la cirugía, y estos cambios pueden persistir semanas después de la operación ${ }^{3}$.

Atelectasia deriva de las palabras griegas ateles y ektasis, que significa expansión incompleta. La atelectasia es un colapso pulmonar con pérdida del volumen pulmonar, ya sea una parte o la totalidad de un pulmón. Pueden deberse a mecanismos de obstrucción/compresión de la vía aérea o por reabsorción al administrar fracciones inspiradas de oxigeno elevadas y a la pérdida del surfactante que favorece los fenómenos de apertura y cierre alveolar cíclico ${ }^{4,5}$. En algunos pacientes la presencia de atelectasias podría ser una de las principales causas de daño pulmonar posquirúrgi$\mathrm{CO}^{6,7}$. Ante la presencia de una atelectasia son varias las situaciones que inducen daño, en primer lugar, los alvéolos que rodean la atelectasia se ven sometidos a fuerzas de tracción de alta energía como consecuencia de la distribución desigual de las fuerzas; dicha energía es transmitida al epitelio capilar generando aumento de la permeabilidad, edema y liberación de proteínas que inactivan más surfactante. El principal determinante para este evento es el volumen corriente, que genera incrementos de la presión transpulmonar en las unidades alveolares vecinas a las zonas de atelectasia. Otra explicación es la desigual entrega de energía en el sistema pulmonar, por la distribución del volumen de manera no uniforme, que tiene como consecuencia áreas de sobredistensión asociado a áreas de colapso y reapertura que perpetúan el ciclo de daño $0^{8,9}$.

Podemos resumir al decir que la VM induce al proceso inflamatorio desde dos aspectos, uno mecánico y otro biológico. Por un lado, se somete al alvéolo a volúmenes y presiones que pueden ser injuriantes comprometiendo la integridad del epitelio alveolar con el consecuente edema y ocupación del espacio alveolar ${ }^{10,11}$; por otro lado, el daño biológico hace referencia a la liberación de citoquinas proinflamatorias y cambios en la funcionalidad del endotelio capilar que resulta en la perpetuación del proceso inflamatorio, que una vez instalado repercute a nivel sistémico constituyendo lo que se denomina biotrauma ${ }^{12}$

No son claras las vías por las cuales los eventos mecánicos producen la liberación de citoquinas. Tanto el cierre y reapertura cíclica como las presiones transpulmonares aumentadas generan cambios en el citoesqueleto que iniciarían cascadas de señalización intracelular y consecuentemente liberación de factores proinflamatorios ${ }^{13}$. Una vez iniciado el proceso pulmonar, la actividad proinflamatoria puede repercutir a nivel sistémico, esto se evidencia por el incremento sérico de citoquinas como el factor de necrosis tumoral alfa (TNF-alfa), la interleucina 6 (IL-6) y la interleucina 8 (IL-8) 12,14,15

La mayor prevalencia de disfunciones pulmonares en el posoperatorio de cirugía cardiovascular respecto de otras cirugías parece responder a varias causas, entre ellas el estrés mecánico y el biotrauma consecuente. En este contexto, las estrategias ventilatorias durante el procedimiento y luego del mismo son determinantes de la evolución de los pacientes.

\section{EFECTO CARDIOVASCULAR DE LA VENTILACIÓN MECÁNICA}

El principal cambio producido por la ventilación mecánica es el cambio a presión positiva intratorácica. La magnitud del impacto depende de varios factores como la función miocárdica prequirúrgica, el estado del volumen intravascular, el nivel de presión positiva intratorácica o las patologías valvulares (Tabla 1). La VM produce cambios hemodinámicos, afectando fundamentalmente la precarga y la poscarga ventricular.

La presión positiva intratorácica reduce el retorno venoso y disminuye la diferencia de presión entre el sistema venoso sistémico y la aurícula derecha, consecuentemente reduce la precarga del ventrículo derecho (VD), esto disminuye el volumen de fin de diástole y del volumen minuto cardíaco.

La reducción de la CRF y las atelectasias presentes en el paciente sometido a cirugía cardiovascular generan una disminución del volumen pulmonar al final de espiración y vasoconstricción hipóxica generando un aumento de la resistencia vascular pulmonar, esto ocasiona el aumento de la poscarga del VD. Para disminuir el impacto de estos cambios, la aplicación de presión positiva de fin de espiración (PEEP) es fundamental como estrategia de ventilación mecánica. E objetivo es evitar el colapso alveolar y mejorar la oxigenación que resultará en la optimización del gasto cardíaco ${ }^{16,19}$

El ventrículo izquierdo (VI) sufre una disminución del volumen de fin de diástole por la caída del gasto cardíaco derecho, pero la poscarga del VI se reduce por acción de la PEEP, ya que disminuye su presión transmural mejorando el gasto cardíaco izquierdo. 
Tabla 2. Modificaciones que produce el uso de PEEP ideal (óptima) y valores de PEEP en exceso. PEEP: presión positiva en fin de espiración. VI: ventrículo izquierdo.

\begin{tabular}{lll} 
& PEEP óptima & PEEP en exceso \\
\hline Presión intratorácica & Incrementa & Incrementa \\
\hline Capacidad residual funcional & Incrementa & Incrementa \\
\hline Compliance pulmonar & Incrementa & Disminuye \\
\hline $\mathrm{PaCO}_{2}$ & Disminuye & Incrementa \\
\hline Shunt & Disminuye & Incrementa \\
\hline Espacio muerto & Disminuye & Incrementa \\
\hline Gasto cardíaco & Incrementa & Disminuye \\
\hline Resistencia vascular pulmonar & Normal & Incrementa \\
\hline Poscarga del VI & Disminuye & Disminuye
\end{tabular}

Durante la inspiración, el aumento del volumen pulmonar comprime la sangre fuera del lecho pulmonar, aumentando la precarga del VI. La combinación de aumento de la precarga y la disminución de la poscarga del VI durante la inspiración resulta en un incremento del volumen sistólico del VI y la presión arterial sistólica durante la ventilación mecánica ${ }^{20}$.

En contraposición a esto la VM produce significativa hipotensión en aquellos pacientes con disminución del volumen intravascular (hipovolemia), la presión positiva intratorácica tendrá mayor impacto en la precarga del VD y consecuentemente en la caída del volumen de fin de diástole del $\mathrm{V}^{21-24}$. Por ello, se debe asegurar un volumen intravascular adecuado y además como ya se ha mencionado, utilizar una estrategia ventilatoria que evite el colapso alveolar y la vasoconstricción hipóxica. Un óptimo gasto cardíaco derecho, que garantice un adecuado volumen de fin de diástole del VI, con la concomitante disminución de la poscarga del VI generada por la presencia de presión positiva intratorácica son los objetivos fundamentales en el paciente sometido a cirugía cardiovascular.

Durante el destete de la VM, cuando el paciente comienza con esfuerzos inspiratorios, la presión intratorácica negativa produce un aumento en la poscarga del Vl, y el aumento previo en el volumen de eyección del VI se pierde.

Normalmente, el gasto cardíaco aumenta en respuesta a un aumento en la demanda metabólica. Si esta respuesta está limitada, un aumento en el consumo metabólico de oxígeno puede causar una reducción en la saturación de oxígeno venoso mixto y favorecer la hipoxemia, y en algunos pacientes, puede provocar isquemia miocárdica y causar fallo del destete ${ }^{25}$.

\section{CIRCULACIÓN EXTRACORPÓREA}

La CEC es un sistema artificial de soporte hemodinámico y ventilatorio temporal durante la parada cardíaca, necesaria para realizar muchas intervenciones. Está constituida por un oxigenador, una bomba y un sistema de catéteres. El contacto con el material extraño y el sistema de bombeo produce con gran frecuencia un estado de inflamación generalizada. Se ha establecido la relación entre la CEC y la vasoplejía posterior, sin embargo, existen resultados controversiales ${ }^{26}$. Ascione et al. demostraron una menor producción de citoquinas proinflamatorias en pacientes sometidos a cirugía cardiovascular sin CEC respecto de las cirugías con $\mathrm{CEC}^{27}$. Se ha documentado también una menor incidencia de complicaciones pulmonares, extubación precoz y menor tiempo de VM ${ }^{28}$. Se puede concluir que el uso de CEC se asocia a la generación de inflamación y potencia los efectos de la VM a presión positiva.

\section{TERAPÉUTICAS PARA MINIMIZAR LA LESIÓN PULMONAR: ESTRATEGIAS DE PULMÓN ABIERTO (OPEN LUNG).}

Esta modalidad ventilatoria utilizada en los pacientes con SDRA tiene como objetivo disminuir la cantidad de energía entregada al sistema utilizando bajos volúmenes corrientes con la asociación de valores de PEEP suficientes para evitar la apertura y cierre cíclico en áreas alveolares inestables. Para un uso óptimo requiere la implementación concomitantemente de maniobras de reclutamiento, esta aproximación se considera actualmente como una de las mejoras estrategias ventilatorias para el SDRA ${ }^{29,30}$. Los objetivos son alcanzar parámetros gasométricos apropiados $\left(\mathrm{pH}>7,20 ; \mathrm{PaO}_{2}>65 \mathrm{mmHg}\right.$ ) en contexto de una PEEP que logre contrarrestar el colapso alveolar por la disminución de la compliance pulmonar. Es fundamental mantener presiones transpulmonares que no sean lesivas, esto se consigue evitando la presencia de una gran amplitud entre la presión meseta y la PEEP (driving pressure $<16 \mathrm{cmH}_{2} \mathrm{O}$ ), además de evitar generar sobredistensión alveolar (valores de presión meseta $<28 \mathrm{cmH}_{2} \mathrm{O}$ ) ${ }^{31}$. Para lograr estos objetivos es necesario la utilización de volúmenes corrientes de 6 ml kg/peso teórico o menores si la presión meseta es elevada. La realización de maniobras de reclutamiento busca la apertura de unidades colapsadas y permite la titulación de un valor de PEEP que evite el colapso espiratorio con mínimo efecto hemodinámico ${ }^{32,33}$. El uso sistemático de maniobras de reclutamiento ha caído en desuso en los últimos tiempos por falta de evidencia en la relación beneficios y daño inducidos por las maniobras de reclutamiento ${ }^{34,35}$. Las maniobras de reclutamiento consisten en la aplicación transitoria (minutos) de altas presiones inspiratorias y representan un riesgo potencial de barotrauma, o trastornos hemodinámicos deletéreos ${ }^{33,36,37}$. Sin embargo, su utilización en el contexto de las cirugías cardíacas se sustenta principalmente para revertir las atelectasias. Amato et al. desarrollaron un protocolo con maniobras de reclutamiento en pacientes sometidos a cirugía cardiovascular. Este estudio prospectivo con asignación aleatoria evaluó la eficacia de una maniobra de reclutamiento intensiva vs un reclutamiento moderado, con resultados favorables para las maniobras de reclutamiento intensivas respecto a complicaciones pulmonares postoperatorias, días de ventilación mecánica y el tiempo de hospitalización ${ }^{38}$.

En la práctica diaria establecer el valor exacto de PEEP, no está aclarado. Se considera PEEP óptima al valor que genera un menor efecto hemodinámico y permite la mayor cantidad de unidades alveolares estables (mejor compliance, sin colapso espiratorio) (Figura 1). Dichos valores de PEEP pueden establecerse mediante el uso de balón esofágico, por valoración de la compliance o usando tablas de $\mathrm{PEEP} / \mathrm{FiO}_{2}$. Recientemente se ha propuesto el uso de tomografía de impedancia como estrategia para titular la PEEP. Cada clínico deberá evaluar la situación particular e incluso los cambios que en el paciente suceden con la modificación de los volúmenes pulmonares al titular PEEP (Tabla 2). 


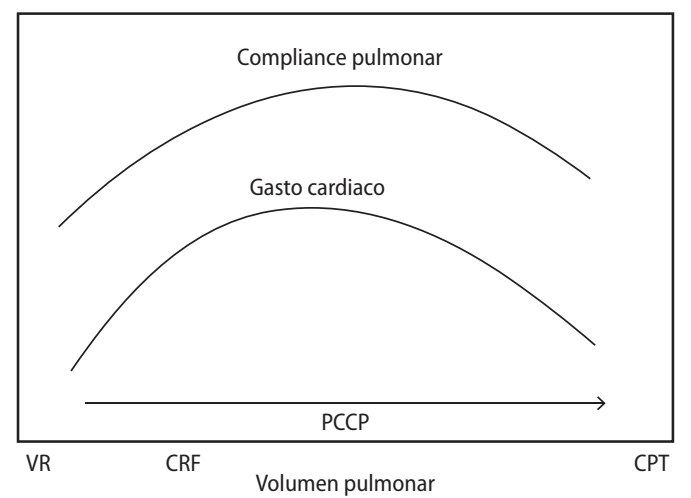

Figura 1. Relación entre PEEP, compliance pulmonar y gasto cardíaco. El incremento en los valores de PEEP aumenta los volúmenes pulmonares, al generar sobredistensión alveolar reducen el gasto cardiaco. El valor de PEEP ideal debería producir la mejor relación entre gasto cardiaco y compliance pulmonar. VR: volumen residual. CRF: capacidad residual pulmonar. CPT: capacidad pulmonar total. PEEP. presión positiva en fin de espiración.

\section{VENTILACIÓN DURANTE CEC}

La hipoventilación durante la CEC genera una mayor incidencia de atelectasias, edema alveolar, disminución de la compliance pulmonar y mayor tasa de infecciones. Existen diversas propuestas para disminuir estas complicaciones ${ }^{39}$. Mantener una presión constante en la vía aérea durante la CEC con presión positiva continua en la vía aérea de $10 \mathrm{~cm} \mathrm{H}_{2} \mathrm{O}$ ha demostrado mejorar el perfil gasométrico en el poso- peratorio ${ }^{40}$. Jhon et al. demostraron que mantener una ventilación a $5 \mathrm{ml} / \mathrm{kg}$ de peso teórico durante la CEC se asocia con una mejor gasometría, un mejor perfil en la mecánica pulmonar y logra menores tiempos de ventilación mecánica y disminución de las complicaciones pulmonares postoperatorias ${ }^{41}$.

\section{CONCLUSIÓN}

La incidencia de trastornos pulmonares y necesidad de ventilación mecánica después de la cirugía cardíaca es frecuente. Los mecanismos implicados en el desarrollo de alteraciones de la mecánica y el intercambio gaseoso pulmonar incluyen dos causas fundamentales: el trauma del procedimiento quirúrgico y el efecto de la ventilación mecánica sobre un entorno inflamatorio, que es mayor en presencia de CEC. La inflamación pulmonar se agrava por la aplicación de VM inadecuada.

Actualmente, se recomienda iniciar la VM con una estrategia de apertura pulmonar temprana en el procedimiento (después de la intubación orotraqueal) con volúmenes corrientes bajos (volumen corriente $6 \mathrm{ml} / \mathrm{kg}$ y uso de PEEP en valores de 8-14 $\mathrm{CmH}_{2} \mathrm{O}$ ), con uso de maniobras de reclutamiento. Si aplicamos estas estrategias podemos esperar una mejora considerable en los parámetros de intercambio de gases, con una menor elevación de los mediadores inflamatorios, una mejor CRF posoperatoria y una menor incidencia de readmisión en la unidad de terapia intensiva. Finalmente, debemos recordar que todo esto se puede lograr con mínimos efectos hemodinámicos adversos.

\section{BIBLIOGRAFÍA}

1. Bardell T, Legare JF, Buth KJ, Hirsch GM, Ali IS. ICU readmission after cardiac surgery. Eur J Cardiothorac Surg 2003;23(3):354-9.

2. Nicholson DJ, Kowalski SE, Hamilton GA, Meyers MP, Serrette C, Duke PC. Post operative pulmonary function in coronary artery bypass graft surgery patients undergoing early tracheal extubation: a comparison between shortterm mechanical ventilation and early extubation. J Cardiothorac Vasc Anesth 2002;16(1):27-31.

3. Weissman C. Pulmonary function after cardiac and thoracic surgery. Anesth Analg 1999;88(6):1272-9

4. Rusca M, Proietti S, Schnyder P, Frascarolo P, Hedenstierna G, Spahn DR, et al. Prevention of atelectasis formation during induction of general anesthesia. Anesth Analg 2003;97(6):1835-9.

5. Magnusson L, Spahn DR. New concepts of atelectasis during general anaesthesia. Br J Anaesth 2003:91(1):61-72.

6. Magnusson L, Zemgulis V, Wicky S, Tyden H, Thelin S, Hedenstierna G. Atelectasis is a major cause of hypoxemia and shunt after cardiopulmonary bypass: an experimental study. Anesthesiology 1997:87(5):1153-63.

7. Verheij J, van Lingen A, Raijmakers $P G$, Spijkstra JJ, Girbes $A R$, Jansen EK, et al. Pulmonary abnormalities after cardiac surgery are better explained by atelectasis than by increased permeability oedema. Acta Anaesthesiol Scand 2005;49(9):1302-10

8. Hubmayr RD. Perspective on lung injury and recruitment: a skeptical look at the opening and collapse story. Am J Respir Crit Care Med 2002:165(12):1647-53.

9. Cressoni M, Chiumello D, Chiurazzi C, Brioni M, Algieri I, Gotti M, et al. Lung inhomogeneities, inflation and [18F]2-fluoro-2-deoxy-D-glucose uptake rate in acute respiratory distress syndrome. Eur Respir J 2016; 47(1):233-42.

10. Meade MO, Cook DJ, Guyatt GH, Slutsky AS, Arabi YM, Cooper DJ, et al. Ventilation strategy using low tidal volumes, recruitment maneuvers, and high positive end-expiratory pressure for acute lung injury and acute respiratory distress syndrome: a randomized controlled trial. JAMA 2008;299(6):637-45.

11. Briel M, Meade M, Mercat A, Brower RG, Talmor D, Walter SD, et al. Higher vs lower positive end-expiratory pressure in patients with acute lung injury and acute

respiratory distress syndrome: systematic review and meta-analysis. JAMA 2010:303(9):865-73.

12. Ranieri VM, Suter PM, Tortorella C, De Tullio R, Dayer JM, Brienza A, et al. Effect of mechanical ventilation on inflammatory mediators in patients with acute respiratory distress syndrome: a randomized controlled trial. JAMA 1999;282(1):54-61.

13. Uhlig U, Fehrenbach H, Lachmann RA, Goldmann T, Lachmann B, Vollmer E, et al. Phosphoinositide 3-OH kinase inhibition prevents ventilation-induced lung cell activation. Am J Respir Crit Care Med 2004;169(2):201-8.

14. Fisher AB, Chien S, Barakat Al, Nerem RM. Endothelial cellular response to altered shear stress. Am J Physiol Lung Cell Mol Physiol 2001;281(3):L529-33.

15. Pinhu L, Whitehead T, Evans T, Griffiths M. Ventilator-associated lung injury. Lancet 2003;361(9354):332-40.

16. Pinsky MR. Cardiovascular issues in respiratory care. Chest 2005:128(5 Suppl 2):592S-5975

17. Dawson CA, Grimm DJ, Linehan JH. Lung inflation and longitudinal distribution of pulmonary vascular resistance during hypoxia. J Appl Physiol Respir Environ Exerc Physiol 1979;47(3):532-6.

18. Reis Miranda D, Gommers D, Struijs A, Meeder H, Schepp R, Hop W, et al. The open lung concept: effects on right ventricular afterload after cardiac surgery. British journal of anaesthesia 2004:93(3):327-32.

19. Agro F, Barzoi G, Doyle DJ, Manieri A. Reduction in pulmonary shunt using the Open Lung Concept. Anaesthesia. 2004;59(6):625-6.

20. Boerlage-van Dijk K, Meregalli PG, Planken RN, Koch KT, Baan J, Jr. Percutane ous left ventricular partitioning device for chronic heart failure. Neth Heart $J$ 2012;20(12):513-515.

21. Pinsky MR. The hemodynamic consequences of mechanical ventilation: an evolving story. Intensive Care Med. 1997;23(5):493-503.

22. Luecke T, Pelosi P. Clinical review: Positive end-expiratory pressure and cardiac out put. Crit Care 2005;9(6):607-21,

23. Jardin F, Dubourg O, Margairaz A, Bourdarias JP. Inspiratory impairment in right ventricular performance during acute asthma. Chest 1987;92(5):789-95. 
24. Jardin F, Farcot JC, Boisante L, Curien N, Margairaz A, Bourdarias JP. Influence of positive end-expiratory pressure on left ventricular performance. N Engl Med 1981;304(7):387-92.

25. Jubran A, Mathru M, Dries D, Tobin MJ. Continuous recordings of mixed venous oxygen saturation during weaning from mechanical ventilation and the ramifications thereof. Am J Respir Crit Care Med 1998;158(6):1763-9.

26. Groeneveld $A B$, Jansen EK, Verheij J. Mechanisms of pulmonary dysfunction after on-pump and off-pump cardiac surgery: a prospective cohort study. J Cardiothorac Surg 2007,2:11.

27. Ascione R, Lloyd CT, Underwood MJ, Lotto AA, Pitsis AA, Angelini GD. Inflammatory response after coronary revascularization with or without cardiopulmonary bypass. Ann Thorac Surg 2000;69(4):1198-204.

28. Al-Ruzzeh S, George S, Bustami M, Wray J, Ilsley C, et al. Effect of off-pump coronary artery bypass surgery on clinical, angiographic, neurocognitive, and quality of life outcomes: randomised controlled trial. BMJ 2006;332(7554):1365.

29. Lachmann B. Open up the lung and keep the lung open. Intensive Care Med 1992; 18(6):319-21.

30. Verbrugge SJ, Sorm V, Lachmann B. Mechanisms of acute respiratory distress syndrome: role of surfactant changes and mechanical ventilation. J Physiol Pharmacol 1997;48(4)537-57.

31. Fan E, Del Sorbo L, Goligher EC, Hodgson CL, Munshi L, Walkey AJ, et al. An Official American Thoracic Society/European Society of Intensive Care Medicine/Society of Critical Care Medicine Clinical Practice Guideline: Mechanical Ventilation in Adult Patients with Acute Respiratory Distress Syndrome. Am J Respir Crit Care Med 2017:195(9):1253-1263.

32. Gattinoni L, Quintel M. How ARDS should be treated. Crit Care. 2016,20:86.

33. Gattinoni L, Tonetti T, Cressoni M, Cadringher P, Herrmann P, Moerer O, et al. Ven- tilator-related causes of lung injury: the mechanical power. Intensive Care Med 2016;42(10):1567-1575

34. Fan E, Checkley W, Stewart TE, Muscedere J, Lesur O, Granton JT, et al. Complications from recruitment maneuvers in patients with acute lung injury: secondary analysis from the lung open ventilation study. Respir Care 2012;57(11):1842-9.

35. Fan E, Wilcox ME, Brower RG, Stewart TE, Mehta S, Lapinsky SE, et al. Recruitment maneuvers for acute lung injury: a systematic review. Am J Respir Crit Care Med 2008; 178(11):1156-63.

36. Cavalcanti $A B$, Suzumura $E A$, Laranjeira $L N$, Paisani DM, Damiani $L P$, Guimarães $H P$, et al. Effect of Lung Recruitment and Titrated Positive End-Expiratory Pressure (PEEP) vs LOW PEEP on Mortality in Patients With Acute Respiratory Distress Syndrome: A Randomized Clinical Trial. JAMA 2017;318(14):1335-1345.

37. Sahetya SK, Brower RG. Lung Recruitment and Titrated PEEP in Mod erate to Severe ARDS: Is the Door Closing on the Open Lung? JAMA2017;318(14):1327-1329.

38. Costa Leme A, Hajjar LA, Volpe MS, Fukushima JT, De Santis Santiago RR, Osawa EA, et al. Effect of Intensive vs Moderate Alveolar Recruitment Strategies Added to Lung-Protective Ventilation on Postoperative Pulmonary Complications: A Randomized Clinical Trial. JAMA 2017:317(14):1422-1432.

39. Magnusson L, Zemgulis V, Tenling A, Wernlund J, Tyden H, Thelin S, et al. Use of a vital capacity maneuver to prevent atelectasis after cardiopulmonary bypass: an experimental study. Anesthesiology 1998;88(1):134-42.

40. Loeckinger A, Kleinsasser A, Lindner KH, Margreiter J, Keller C, Hoermann C. Continuous positive airway pressure at $10 \mathrm{~cm} \mathrm{H(2)O} \mathrm{during} \mathrm{cardiopulmonary} \mathrm{bypass}$ improves postoperative gas exchange. Anesth Analg 2000;91(3):522-7.

41. John LC, Ervine IM. A study assessing the potential benefit of continued ventilation during cardiopulmonary bypass. Interac Cardiovasc Thorac Surg 2008;7(1):14-7. 